Check for updates

Cite this: J. Anal. At. Spectrom., 2019 34,1098

Received 13th December 2018 Accepted 1st March 2019

DOI: $10.1039 / \mathrm{c} 8 \mathrm{ja} 00439 \mathrm{k}$

rsc.li/jaas

\section{Identification of nanoparticles and their localization in algal biofilm by 3D-imaging secondary ion mass spectrometry $\dagger$}

\author{
Pietro Benettoni, ${ }^{a}$ Hryhoriy Stryhanyuk, (D) *a Stephan Wagner, (D) ${ }^{\mathrm{b}}$ Felix Kollmer, (D) \\ Jairo H. Moreno Osorio, (iD ad Matthias Schmidt, (iD a Thorsten Reemtsma (iD be \\ and Hans-Hermann Richnow (iD)
}

Characterization of nanoparticle (NP) interaction with an organic host requires identification and localization of nano-sized phases via high-resolution 3D imaging. Simultaneous molecular and spatial resolution causes many analytical approaches to face inherent performance limits, but this challenge can be overcome with time-of-flight secondary ion mass spectrometry (ToF-SIMS). To implement ToF-SIMS analysis of complex organic systems, a careful sample preparation is required to ensure ultra-high vacuum compatibility and to preserve the native chemical composition and spatial arrangement. In this present study, we developed a new method for the deposition of NPs from suspensions employing a polymer-coated substrate to avoid NP aggregation and to eliminate dissolved contaminants. We achieved a lateral resolution of $108 \mathrm{~nm}$ simultaneously with a mass resolving power $(M R P=M / \Delta M)$ above 5000 in delayed extraction mode. Optimized ToF-SIMS performance allowed for (i) 3D localization of $\mathrm{TiO}_{2} \mathrm{NPs}$ in Chlorella vulgaris biofilm, (ii) precise isotope-pattern-based identification of $\mathrm{TiO}_{2}$-related secondary ions and (iii) elucidation of their composition dependence on the nearest molecular surrounding of the NPs. The developed sample preparation method in combination with the high resolution 3D visualization of NPs in algal biofilm paves the way to study NP fate and localization in complex environments simultaneously.

\section{Introduction}

Nanotechnology has emerged as one of the major fields that have the potential to remodel many aspects of our society including medicine, engineering, nutrition and many others. However, the extensive use of nanoparticles in our everyday life

\footnotetext{
${ }^{a}$ Department of Isotope Biogeochemistry, Helmholtz Centre for Environmental Research - UFZ, Permoserstraße 15, 04318 Leipzig, Germany. E-mail: gregory. stryhanyuk@ufz.de

${ }^{b}$ Department of Analytical Chemistry, Helmholtz Centre for Environmental Research UFZ, Permoserstraße 15, 04318 Leipzig, Germany

'IONTOF GmbH, Heisenbergstraße 15, 48149 Münster, Germany

${ }^{d}$ Department of Civil and Mechanical Engineering, University of Cassino and Southern Lazio, via Di Biasio 43, 03043 Cassino, Italy

'Institute of Analytical Chemistry, University of Leipzig, Johannisallee 29, 04103 Leipzig, Germany

$\dagger$ Electronic supplementary information (ESI) available: Experimental section describing methods of microscopy and DLS, structure and achieved thickness of Mowital polymer, SEM images of NPs before and after cleaning employing the newly developed method, DLS distribution of $\mathrm{TiO}_{2} \mathrm{NPs}$ suspension in water, lateral resolution statistics, microscopy images of $C$. vulgaris before and after incubation with NPs, mass spectra of C. vulgaris biofilm exposed to $\mathrm{TiO}_{2} \mathrm{NPs}$ and non-exposed biofilm, RGB overlays of the 4 different Ti containing ions, 3D image with $\mathrm{XZ}$ cross section, linescan over $\mathrm{PO}_{3}{ }^{-}$secondary ion map. See DOI: 10.1039/c8ja00439k
}

is associated with potential risks towards humans and the environment. ${ }^{1,2}$ In fact, the interaction between nanoparticles (NPs) and a biological matrix is yet to be fully understood..$^{3,4}$ To address this issue, it is necessary to characterize NPs applying a comprehensive analysis of size, shape, chemical composition, surface properties, stability and protein corona formation within a complex biological system. ${ }^{5}$ Therefore, sensitive and versatile methods are required to spatially resolve and chemically identify the nanomaterials in complex biological media. ${ }^{4}$ The application of secondary ion mass spectrometry (SIMS) in life science ${ }^{6,7}$ is a promising technique for the characterization of NPs in biological media. In fact, its sensitivity, high spatial resolution and mass resolution offer label-free chemical 3D imaging capabilities down to the nanometer scale. ${ }^{7-9}$ SIMS analyses involving identification and localization of NPs have already been reported in previous studies., ${ }^{5,9-11}$

State-of-the-art Time-of-flight (ToF)-SIMS instruments provide the advantage of using cluster ion sources for molecular $3 \mathrm{D}$ imaging of complex biological samples. ${ }^{12,13}$ Another advantage of using ToF-SIMS for biological sample analysis is the charge compensation implemented with flooding e-gun in combination with flooding gas. Gas flooding prevents the molecular structure damage upon direct irradiation of organic sample with flooding electrons and provides charge carriers of 
both polarities $\left(\mathrm{Ar}^{+}\right.$and secondary $\left.\mathrm{e}^{-}\right)$for charge compensation. Furthermore, ToF-SIMS analyzer allows for the detection of all secondary ion species within a broad range of $\mathrm{m} / \mathrm{z}$ ratio up to $\sim 1000$ without a limitation of the number of the detected species. ${ }^{\mathbf{1 4}}$ However, the time-of-flight analyzer requires a short primary ion pulse width for high mass resolution. This reduces the achievable duty cycle and leads to low primary currents. The common approach is to compress a longer primary ion pulse by axial bunching to the sub ns regime in order to combine high mass resolution with appropriate primary ion currents. The compression of the primary ion bunch enhances the mass resolution but causes a broadening of the primary ion energy distribution and therefore reduces the lateral resolution by chromatic aberration. The loss of lateral resolution upon the improvement of the mass resolution by ion bunch compression leads to a trade-off between lateral and mass resolutions (i.e. one is achieved at the cost of the other) in the common approach. ${ }^{7,9,15}$ However, high lateral resolution is required for the precise localization of a nano-sized phase in a host. At the same time, high mass resolution is necessary to resolve different secondary ion species and to identify them accurately. Thus, both lateral and mass resolutions are required for analysis of NP fate within a complex organic matrix or an organism..$^{2,16-19}$

In order to achieve simultaneous high mass and lateral resolution with ToF-SIMS, the mass resolution has to be decoupled from the duration of the primary ion pulse. Such decoupling has already been implemented with pulsed extraction field, delaying the secondary ion extraction until all secondary ions have left the sample surface. The delayed extraction mode of ToF-SIMS operation was initially developed for analysis of samples with strong topography. ${ }^{20}$ It allows for simultaneous lateral and mass resolution and has been demonstrated by Vanbellingen et al. to deliver $400 \mathrm{~nm}$ lateral resolution upon imaging Purkinje cells of $20 \mu \mathrm{m}$ average size. ${ }^{15}$ More recently, Veith et al. reported an achieved lateral resolution of $673 \mathrm{~nm}$ simultaneously with a mass resolution of 4000 upon analysis of lung tissue section. ${ }^{21}$ It is obvious that the reported performances in terms of lateral resolution and mass resolution are distinctly reduced compared with standard modes under static extraction conditions. This is caused by the fact that the delayed extraction mode is especially challenging for non-conductive samples with strong topography. Furthermore, the reported lateral resolution is not sufficient for chemical imaging of single NPs.

In the present study, we aimed at increasing the lateral resolution of ToF-SIMS instrument below $200 \mathrm{~nm}$ maintaining sufficient secondary ion yield and mass resolving power for chemical identification of NPs and their localization in biological host. Moreover, we have elaborated the methods for (i) representative deposition of NPs from suspension employing polymer-coated substrate and (ii) dehydration of biological cells required by Ultra-High Vacuum (UHV) sample environment of ToF-SIMS. The developed sample preparation techniques allow: (i) to preserve chemical identity of the NPs and biological host constituents; (ii) to minimize the distortion of sample morphology (e.g. cell shrinkage, blasting, swelling, crystallization of salt residuals, aggregation of NPs or single cells etc.). The quality of sample preparation has been validated with scanning electron microscopy (SEM) and ToF-SIMS analysis of $\mathrm{TiO}_{2}$ NPs as deposited from suspension and by their 3D imaging in algal biofilm. With the performance of ToF-SIMS instrument in the optimized delayed extraction mode, a lateral resolution in the $100 \mathrm{~nm}$ range was achieved simultaneously with the mass resolving power above 5000, allowing identification and localization of nano-sized phases and organic host compartments.

\section{Material and methods}

\section{Preparation of sample carrier}

Conductive Si wafers of $10 \times 10 \mathrm{~mm}^{2}$ size (Plano GmbH, G339010 , Boron-doped) were used as sample supporting plates. The wafers were chemically cleaned in an ultrasonic bath (Sonorex Digitec, Bandelin electronic, Berlin, Germany) for $15 \mathrm{~min}$ in absolute ethanol (Chemsolute, 2246, Th. Geyer). Afterwards, they were treated with UV-Ozone cleaner (PULC-120, UFZ Leipzig) ${ }^{22}$ to remove residual organic material remaining after chemical treatment. ${ }^{23}$ The efficiency of the cleaning was confirmed via contact angle observation.

The cleaned wafers were coated with a solution of polyvinyl butyral called Mowital B $30 \mathrm{~T}$ (Kuraray Europe GmbH) that was prepared as follows: $2 \mathrm{~g}$ of Mowital B $30 \mathrm{~T}$ powder were dissolved in $40 \mathrm{~mL}$ of diacetone alcohol (4-hydroxy-4-methyl-2pentanone, Sigma-Aldrich, H41544) in order to obtain a $5 \mathrm{wt} \%$ solution. The bottle with the solution was placed horizontally on a shaker (Multi-functional Orbital Shaker PSU-20i, Biosan, Riga, Latvia) for 5 days with an orbital speed of $245 \mathrm{rpm}$ (reciprocal and vibro parameters kept off). After complete dissolution, the bottle was placed for 2 days in the dark to release the trapped gas phase bubbles.

The $300 \mathrm{~nm}$ thickness of polymer coating layer was achieved by injecting $200 \mu \mathrm{L}$ of dissolved Mowital polymer onto the silicon wafer rotating at $60 \mathrm{rps}$ in a spin coater (SCI Series, LOTQuantumDesign GmbH, Darmstadt, Germany) and stopping the rotation after $\approx 2 \mathrm{~min}$. The thickness of polymer layer was measured using a non-contact 3D surface profiler (PLu Neox, Sensofar, Barcelona, Spain).

\section{Treatment and deposition of nanoparticles}

$2.5 \mathrm{mg}$ of $70 \mathrm{~nm} \mathrm{TiO}_{2}$ NPs (National Institute of Standards and Technology, NIST) were dispersed with $5 \mathrm{~mL}$ of Milli-Q (MQ) water to obtain the NP suspension. The suspension was sonicated for $10 \mathrm{~min}$ in an ultrasonic bath (Transsonic TS 540, Elma $\mathrm{GmbH}$, Singen, Germany) to assure the complete dispersion of the NPs. The $5 \mathrm{~mL}$ suspension was further diluted with $45 \mathrm{~mL}$ of

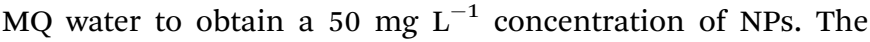
resulted $50 \mathrm{~mL}$ suspension was sonicated with a vial tweeter (UP200St, Hielscher Ultrasonics GmbH, Teltow, Germany) for $5 \mathrm{~min}$.

$\mathrm{Au}$ NPs of $200 \mathrm{~nm}$ size (British Biocell International, BBI) were prepared for the optimization and correction of possible lateral drift in ToF-SIMS experiments. $0.5 \mathrm{~mL}$ of $50 \mathrm{mg} \mathrm{L}^{-1} \mathrm{Au}$ 
NP suspended in water were centrifuged (5424 R centrifuge with FA-45-24-11 rotor, Eppendorf GmbH, Germany) at $10000 \mathrm{rpm}$ for $6 \mathrm{~min}$. Afterwards, the supernatant was removed and the remaining NPs were resuspended in $0.5 \mathrm{~mL}$ of MQ water. This procedure was repeated twice in order to remove the dissolved $\mathrm{KCl}$ and $\mathrm{NaCl}$ salt contaminants from the suspension.

Another method for cleaning of a NP suspension from soluble contaminants was developed to avoid a possible loss of suspended material during supernatant removal after centrifugation. For this purpose we have elaborated an ad hoc deposition method and an appropriate centrifuge adaptor to host samples of different geometries. After depositing a $5 \mu \mathrm{L}$ drop of NP suspension onto a freshly prepared polymer surface, the sample carrier was centrifuged at $1000 \mathrm{rpm}$ for 3 min (ROTINA 380R, swing out rotor 1798 , Hettich $\mathrm{GmbH} \&$ CO. KG, Germany).

\section{Growth and preparation of algal cells}

Chlorella vulgaris ACUF_809 green microalgae (http:// www.acuf.net) was isolated from a pilot scale moving bed biofilm reactor during a nitrification process of industrial wastewater. For the growth of stock algae culture cells the original Bold's basal medium (BBM) ${ }^{24}$ was modified by increasing the concentration of $\mathrm{KH}_{2} \mathrm{PO}_{4}$ and $\mathrm{K}_{2} \mathrm{HPO}_{4}$ up to $175 \mathrm{mg} \mathrm{L}{ }^{-1}$.

Silicon wafers coated with $300 \mathrm{~nm}$ layer of Mowital B $30 \mathrm{~T}$ polymer as described above, were used as carriers for microalgae biofilm growth. Freshly cleaned and polymer-coated wafers were immobilized on a fine agarose base $(1 \mathrm{~mL}$ of $10 \mathrm{wt} \%$ agarose; SeaKem LE, Lonza, USA) in $\varnothing 30 \times 15 \mathrm{~mm}$ plastic Petri dishes. Afterwards, the dishes were filled with $3 \mathrm{~mL}$ of the growth medium (modified BBM) containing the C. vulgaris cells $\left(2.5 \times 10^{6}\right.$ cells per $\left.\mathrm{mL}\right)$ in exponential growth phase. The initial cell density was determined with a calibration curve that relates the absorbance at $680 \mathrm{~nm}$ and the biomass concentration. $^{25}$ The algal cells were incubated in the Petri dishes placed on an orbital shaker (Compact Shaker KS 15 A, Edmund Buehler, Bodelshausen, Germany) at $60 \mathrm{rpm}$ upon illumination (array of 5 linear white fluorescent lamps T8 Polylux XLR, GE Lighting, Frankfurt, Germany) in constant day/ night rhythm $(14 \mathrm{~h} / 10 \mathrm{~h}$ of illumination $\mathrm{ON}-\mathrm{OFF})$ at $20 \pm 3{ }^{\circ} \mathrm{C}$.

On the $19^{\text {th }}$ day of incubation, the growth medium was substituted with a freshly prepared one containing $\mathrm{TiO}_{2} \mathrm{NPs}^{(1}$ $\mu \mathrm{g} \mathrm{mL}^{-1}$ ) and the incubation continued upon constant shaking to maintain a homogeneous NP distribution in the growth medium and to enhance the frequency of NP-cell collision events. $^{\mathbf{2 6}}$

After $24 \mathrm{~h}$ of incubation in the NP-containing growth medium, the wafers with algal biofilm were carefully removed from the Petri dish and the cells were fixed upon drying for $2 \mathrm{~h}$ in the fixative vapors of paraformaldehyde-ethanol-water mixture (10-37-53 vol\%) maintained under a $0.5 \mathrm{~L}$ glass bell on a ceramic heating plate (IKA C-MAG HP 10, IKA-Werke, Staufen, Germany) at $40{ }^{\circ} \mathrm{C}$. After complete drying of algal biofilm, the carrier wafers were moved out from the fixative vapors and placed in a desiccator overnight.

\section{ToF-SIMS analysis}

The NPs deposited from the suspension on the polymer-coated carriers and the samples of chemically fixed algal biofilm were analyzed with a TOF-SIMS.5 instrument (IONTOF GmbH, Münster, Germany). ToF-SIMS measurements were performed in delayed extraction mode providing mass resolving power (MRP) within 3000-7000 range and the lateral resolution of about $110 \mathrm{~nm}$.

As primary projectiles $\mathrm{Bi}_{3}{ }^{+}$cluster ions of $30 \mathrm{keV}$ energy from liquid metal ion gun (LMIG NanoProbe, mounted at $45^{\circ}$ to the analyzer axis) were employed for the analysis of NPs on the polymer-coated carriers and in the algal biofilm. The highest lateral resolution is achieved by $\mathrm{Bi}_{3}{ }^{++}$primary ion species but $\mathrm{Bi}_{3}{ }^{+}$offers a higher ion current and higher secondary ion yield for the desired secondary ion species. The $105 \mu$ s repetition period of primary ion pulse delivered $0.05 \mathrm{pA}$ current of $\mathrm{Bi}_{3}{ }^{+}$and allowed the detection of secondary ions within range of 10-3500 $\mathrm{m} / \mathrm{z}$ (mass-to-charge ratio). The analyzer extraction voltage was reduced to $1 \mathrm{kV}$ and the energy was increased to $3 \mathrm{keV}$ as compared to the standard settings in order to increase mass resolution by decreasing the relative energy spread of the secondary ions. The analysis was performed by rastering the primary ion beam randomly in $512 \times 512$ pixels over $60 \times 60$ $\mu \mathrm{m}^{2}$ sample area. After each primary beam shot, the sample charging was compensated with $16 \mathrm{eV}$ electrons from flooding e-gun (FEG) and Ar gas injection system (Ar-GIS) maintaining the partial Ar gas pressure within $4 \times 10^{-7}$ to $2 \times 10^{-6}$ mbar in the analysis chamber.

The data were acquired in frames. Each data frame was generated after 10 scans over the analysis area with one single shot per each pixel. Two different sputter ion sources were applied in the reported experiments: (i) $500 \mathrm{eV} \mathrm{Cs}^{+}$ion source and (ii) $5 \mathrm{keV}[\mathrm{Ar}]_{1750}$ gas cluster ion beam (GCIB-source) for sample sputtering in non-interlaced mode.

The lateral resolution of ToF-SIMS was checked on a Chessy test specimen (Agar Scientific Ltd, UK) by random rastering an area of $20 \times 20 \mu^{2}$ with $1024 \times 1024$ pixels and single shots per pixel. The pixel size was $19.5 \mathrm{~nm}$. For this measurement, $\mathrm{Bi}_{3}{ }^{+}$cluster ions were also used as primary projectiles for analysis and $\mathrm{Cs}^{+} 250 \mathrm{eV}$ for sputtering to enhance the yield of negative secondary ions (from $\mathrm{Si}$ and $\mathrm{Au}$ ). In total, 150 scans were acquired on the test specimen and 115 single scans were used for the lateral shift correction based on $\mathrm{Au}_{3}{ }^{-}$signal over the whole analyzed area.

Data treatment was performed using SurfaceLab 6.7 software (IONTOF GmbH). Lateral shift correction and mass shift correction were applied during the reconstruction of the acquired data frames. For the analysis of NPs on the polymercoated carriers, $\mathrm{Au}^{-}$and $\mathrm{Ti}^{+}$signals over the whole analyzed area were used for lateral drift correction of $\mathrm{Au}$ NPs and $\mathrm{TiO}_{2}$ NPs respectively. The lateral shift of the data acquired upon analysis of algal biofilm was corrected by the total ion signal over the entire analysis area. Lateral corrections were implemented on single scans with "quadruple precision" in order improve the accuracy of the correction. For "quadruple precision" each pixel is up-scaled to $4 \times 4$ pixels and the shift values 
are calculated using the high resolution data. The high resolution results are then downsampled to the original pixel resolution. Finally, the data are corrected using these integral pixel shifts.

\section{Results and discussion}

\section{Optimization of sample carrier}

Well defined elemental composition, adjustable surface properties, ease of treatment and handling defined our choice of Siwafer as sample supporting plate for ToF-SIMS analysis. However, the native properties of a surface are always affected by organic molecules from surroundings. Even after careful chemical cleaning, residuals from cleaning agents remain and molecules of volatile substances adhere on the surface. The adsorbed molecules modify the pristine chemical and physical properties of the surface e.g., alter the surface hydrophilicity. This adsorption occurs to some extent even if the surface is kept in a sterile environment or in ultra-high vacuum (UHV) where residual gas molecules and dust particles are present. ${ }^{27,28}$ Therefore, after chemical cleaning, a further UV-Ozone treatment was applied to remove residual organic molecules ${ }^{23}$ and recover the native properties of the Si-wafer surface before coating it with a polymer.

Silicon wafers were coated with polymer to tune their surface polarity depending on the properties of the analyte to be deposited. Polymers of the Mowital family offering different degree of viscosity, mechanical hardness and surface polarity were used for carrier coating in the present study. With 5 wt $\%$ solution of Mowital B $30 \mathrm{~T}$ polymer (Fig. S-1A $\dagger$ ) a $\approx 300 \mathrm{~nm}$ thick layer was achieved via spin coating (Fig. S-1B $\dagger$ ). The polymercoated carrier allowed us to avoid aggregation of deposited NPs during drying and to mimic an organic host. Furthermore, a sticky polymer layer prevents detachment, relocation or removal of small-size NP fractions when hit by the ion beam. Thus, it maintained the sample integrity and extended the availability of NPs (i.e. efficient detection of NPs related secondary ions species) during the ToF-SIMS analysis.

\section{Deposition of NP suspensions onto the polymer-coated sample carrier}

The main challenges of NP deposition onto a carrier are (i) to obtain representative single-particle distribution (i.e. avoid aggregation) and (ii) to preserve their chemical composition. In fact, preliminary SEM-EDX analyses showed the presence of $\mathrm{KCl}$ and $\mathrm{NaCl}$ residuals dissolved in the suspension of Au NPs causing a strong aggregation of the NPs around salt crystals after drying (Fig. S-2A and $\mathrm{B} \dagger$ ). Even if the NP suspension is free of soluble contaminants, the hydrophobicity of a carrier surface may cause aggregation of NPs at the rim of the suspension droplet upon drying. This aggregation process is called the coffee-ring effect. ${ }^{29-32}$

Centrifugation, followed by supernatant removal, might be one option to remove dissolved contaminants from the suspension. However, it is not always the optimal method since the supernatant removal may cause the loss of a fraction of NPs that are not included into the pellet upon centrifugation.

To reduce the risk of analyte loss during separation of NP and dissolved contaminants, a new method was developed for the deposition of NP from suspensions. The suggested method employs a system comprising a Si wafer coated with a hydrophilic polymer layer (e.g. Mowital B $30 \mathrm{~T}$ ). The hydrophilicity of the polymer coating layer is a key feature of this method that facilitates the transfer of solutes from the top to the bottom of the polymer layer. The transfer of the soluble fraction is enhanced via centrifugation with the centrifugal force orthogonal to the sample carrier plane (Fig. 1). Another advantage is the possibility of consecutive depositions and washings. After each centrifugation step, an additional amount of an analyte suspension can be deposited over the previous one and washed.

The accumulation of NPs via consecutive depositions onto the sample carrier avoids the necessity of using highly concentrated suspensions where the risk of NP aggregation and sedimentation increases. Furthermore, a thorough removal of soluble contaminants and a gain in the analyte concentration on the polymer surface may help to avoid interferences, overcome saturation issues and detection limits of analytical techniques.

SEM analysis demonstrates that after the centrifugation of the suspension droplet on the polymer, the soluble components of the suspension are removed while its insoluble components stay attached to the polymer surface available for analysis (Fig. S-3A and $\mathrm{B}^{\dagger}$ ). Thus, the developed method paves the way for a reproducible deposition of NPs from suspension providing a representative NP distribution over $\sim 1 \mathrm{~mm}^{2}$ area. Such a preparation method helps to avoid the necessity of searching for an optimal analysis field with a complementary visualization technique that are often destructive and may cause the loss of sample fidelity (e.g., modifications and damages induced by laser beam, high-energy ionizing radiation, electron and ion beam probes). This challenge is faced by analytical techniques with moderate lateral resolution where the analysis result is

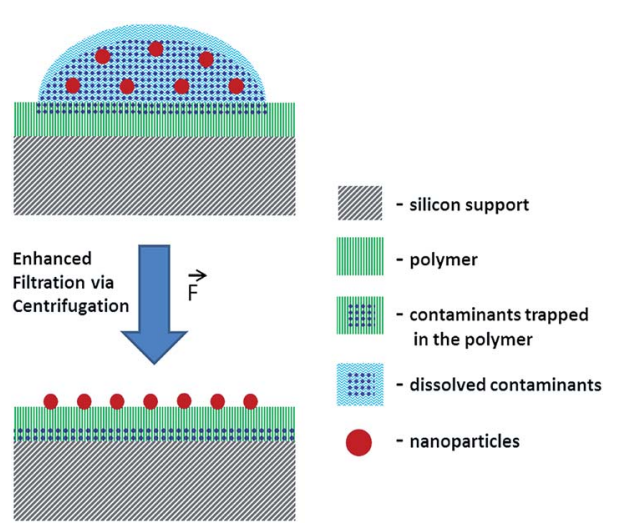

Fig. 1 Sketch of the developed NPs deposition and cleaning concept. After the deposition, the hydrophilicity of the polymer coated silicon wafer allows the penetration of water and solutes into the bottom part of the polymer leaving the clean NPs onto the upper part. This filtration process can be enhanced via centrifugation. 
averaged over relatively large area defined by a probe size (e.g. laser beam spot in laser desorption experiments) exceeding the analyte particle size.

SEM studies of $70 \mathrm{~nm} \mathrm{TiO}_{2}$ NPs deposited on the sample carrier were shown to form aggregates of about $\sim 0.3-1 \mu \mathrm{m}$ in size (Fig. S-3B $\dagger$ ). ${ }^{33-36}$ This aggregation is also known for other types of $\mathrm{NPs}^{37,38}$ and is already occurring in suspension as demonstrated via DLS (Fig. S-4†).

\section{NPs detection and identification with ToF-SIMS}

Au nanoparticles. $200 \mathrm{~nm}$ Au NPs deposited on the polymer layer were analyzed with ToF-SIMS to demonstrate its performance with optimized analytical conditions applied on an insulating sample carrier. Gold is known to be preferably ionized negatively. Therefore, a $500 \mathrm{eV} \mathrm{Cs}^{+}$sputter beam was applied to enhance the yield of negative $\mathrm{Au}^{-}$ions (Fig. 2A). With this measurement the lateral resolution was shown to reach $108 \mathrm{~nm}$ in $Y$-direction (Fig. 2B). It is worth to note that the lateral resolution in horizontal $X$-direction (Fig. 2C) was found to be remarkably different (about $170 \mathrm{~nm}$ ). Such a difference is due to the impact angle of 45 degrees of the primary ion beam. In fact, when impinging the sample at $45^{\circ}$, a circular beam shape results in an elliptically shaped spot stretched by a factor of $\sqrt{2}$ in the $X$ direction. A beam diameter of $108 \mathrm{~nm}$ in $Y$ direction would be distorted to $151 \mathrm{~nm}$ because of this effect only. Additional elongation in the $X$ direction is expected when the measurement is performed across a spherical particle rather than a flat surface. ${ }^{39}$ These two effects are in agreement with our measured values of lateral resolution in $X$ and $Y$ directions.

The lateral resolution achieved on the insulating sample carrier (Fig. 2) is shown to be equal to the one obtained on the conductive standard Chessy structure (Fig. 3) with the optimized ToF-SIMS performance in delayed extraction mode. To further validate this achievement, statistical analyses of linescans on each side of the 50 squares (displayed in Fig. 3A) and on Au NPs in both $x$ and $y$ directions was carried out (box plots are displayed in Fig. $\mathrm{S}-5 \dagger$ ).
$\mathrm{TiO}_{2}$ nanoparticles. Studies on NP interaction with complex matrices demand the identification of low abundance NPs from a suspension often containing organic residuals (e.g. tissue fragments, microorganisms, cells lysate and adsorbed organic molecules). ToF-SIMS analysis of inorganic NPs in such samples requires gentle removal of organic components and efficient detection of NP-related secondary ions. An argon cluster ion beam was reported to sputter the organic compartments ${ }^{\mathbf{4 0}}$ and leaves inorganic sample components accessible for the analyses. Therefore, we employed the sputtering $5 \mathrm{keV}$ argon cluster ion beam for organics removal and the $30 \mathrm{keV} \mathrm{Bi}_{3}{ }^{+}$one for the analysis of $\mathrm{TiO}_{2}$ NPs deposited onto the polymer-coated carrier. The Fig. 4A shows the lateral distribution of the NPs by the combined $\mathrm{Ti}^{+}$and $\mathrm{TiO}^{+}$secondary ion signals. Other detected Ti-containing $\mathrm{Ti}_{x} \mathrm{O}_{y}(x, y>1)$ secondary ion mass peaks (i.e. $\mathrm{Ti}_{2} \mathrm{O}_{2}{ }^{+}, \mathrm{Ti}_{2} \mathrm{O}_{4}{ }^{+} \mathrm{Ti}_{3} \mathrm{O}_{5}{ }^{+}$) showed a relative yield of about 0.1 as compared with $\mathrm{Ti}^{+}$. Fig. 4B shows that the relative intensities of the different $\mathrm{Ti}$ isotope peaks clearly reproduce the $\mathrm{Ti}$ isotopic distribution pattern as compared with the natural Ti isotope abundance (Fig. 4C). This characteristic isotopic pattern of $\mathrm{Ti}$ was later used in this study for the identification of $\mathrm{TiO}_{2} \mathrm{NPs}$ in algal biofilm (C. vulgaris).

\section{ToF-SIMS analysis of $\mathrm{TiO}_{2}$ NPs in algal biofilm}

The optimized ToF-SIMS analysis conditions in delayed extraction mode were then applied for the identification and localization of $\mathrm{TiO}_{2}$ NPs in green microalgae C. vulgaris to demonstrate the applicability of our analytical approach also on complex systems. Freshwater microalgae are an important test organism in aquatic ecotoxicology; ${ }^{\mathbf{4 1}}$ and as an oxygen producer they play an important role in the aquatic ecosystem. ${ }^{34-36}$

The algal biofilm was imaged on the $19^{\text {th }}$ day after inoculation and the typical round shaped $C$. vulgaris cells attached to the polymer were clearly visualized before their exposure to $70 \mathrm{~nm} \mathrm{TiO}_{2}$ NPs (Fig. S-6A $\dagger$ ).

After $24 \mathrm{~h}$ of exposure, the medium containing NPs was removed and the protocol of fixation in vapors ${ }^{32}$ (see Experimental section $\dagger$ ) was applied to stop the exposure of the cells to
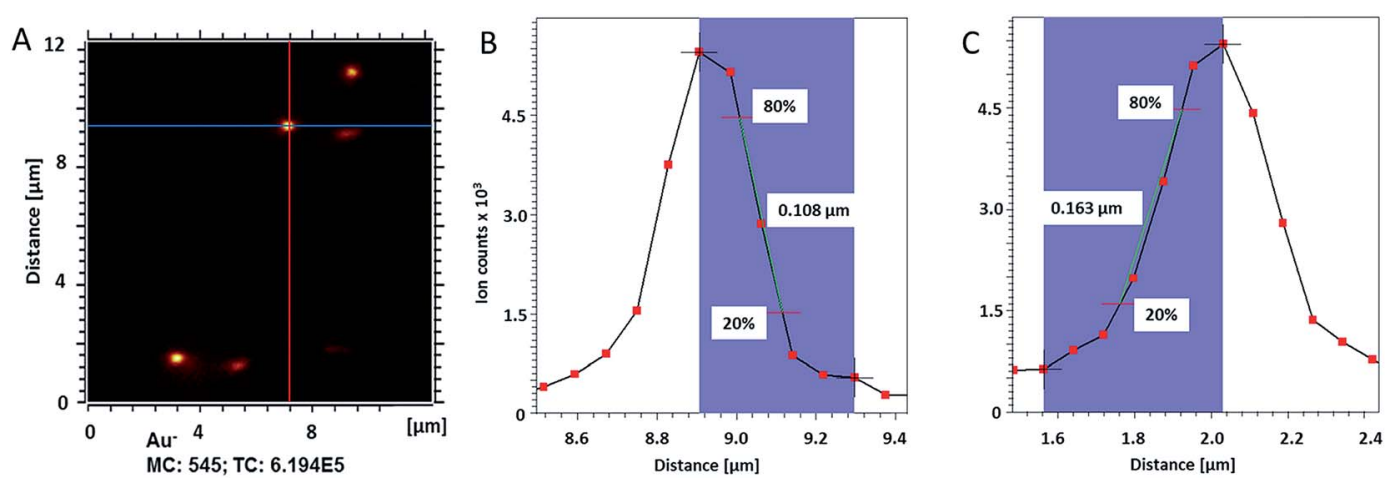

Fig. 2 (A) ToF-SIMS image of $200 \mathrm{~nm}$ Au NPs on insulating sample carrier acquired in delayed extraction mode. Solid lines indicate the position for the determination of the lateral resolution in vertical (red) and horizontal (blue) directions. (B) The linescan in vertical direction ( $Y$-Profile) reveals the lateral resolution of $108 \mathrm{~nm}$ while (C) the linescan in horizontal direction (X-Profile) reveals a resolution of $163 \mathrm{~nm}$. Both determinations were done according to the $80-20 \%$ slope. The lateral resolution measured in $Y$ direction is in agreement with the lateral resolution measured on the standard conductive sample (Fig. 3C). Red squares in (B) and (C) indicate individual pixels. 
A

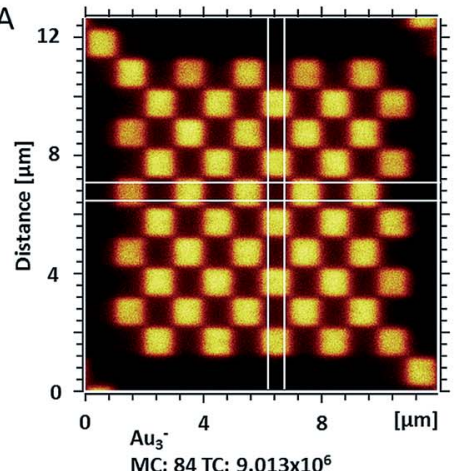

C

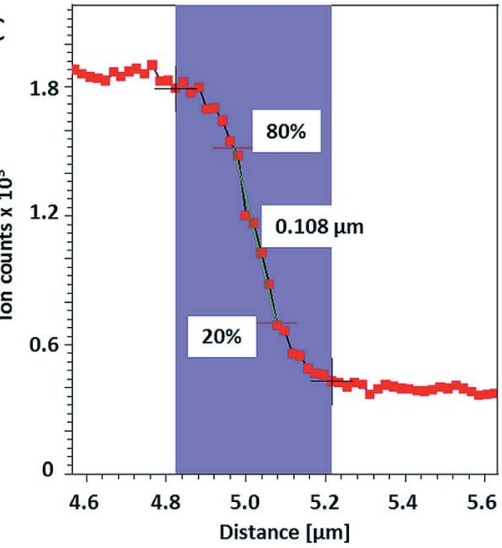

B

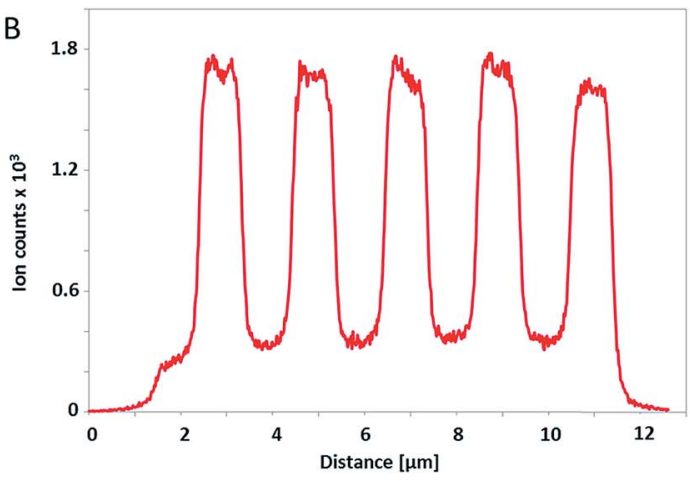

D

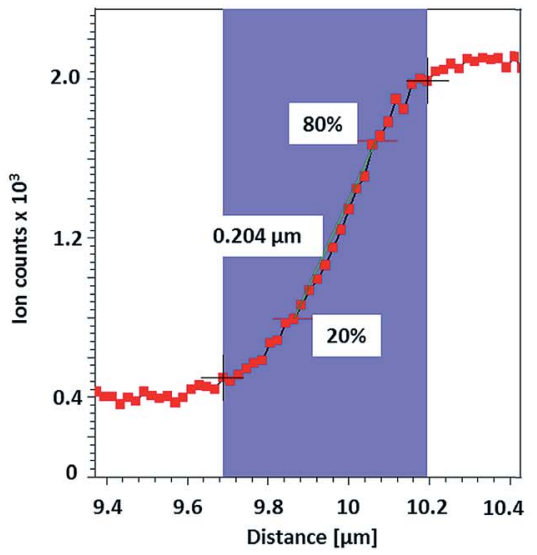

Fig. 3 ToF-SIMS image of standard Chessy structure sample acquired in delayed extraction mode. (A) Checkerboard pattern cropped for clarity. In this test specimen, the smallest square has a size of $1 \mu \mathrm{m}$. White solid lines indicate the regions for the determination of the lateral resolution. (B) Overall lateral profile of the area integrated between white vertical lines. Lateral profiles (C) in vertical direction revealing a resolution of $108 \mathrm{~nm}$ and (D) in horizontal direction revealing a resolution of $204 \mathrm{~nm}$ according to the $80-20 \%$ slope.
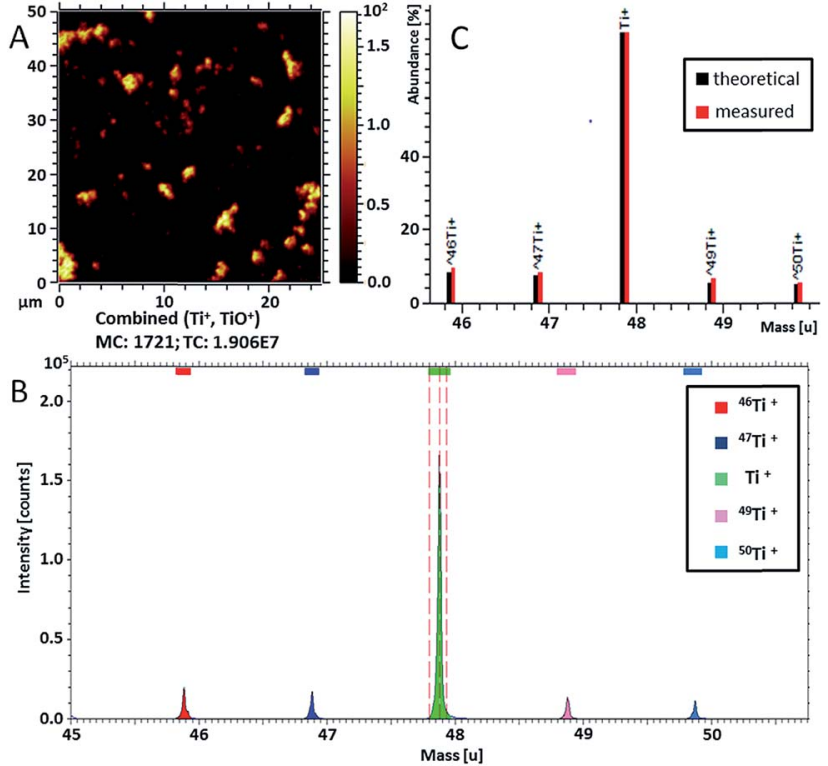

Fig. 4 Results of ToF-SIMS analysis of $\mathrm{TiO}_{2}$ NPs in delayed extraction mode. (A) Lateral yield distribution overlay of representative Ti-containing secondary ion species. (B) Measured isotopic distribution of $\mathrm{Ti}^{+}$, all isotopes of titanium are clearly visible in the spectra and are in agreement with the natural abundance pattern as shown in (C). cytotoxic $\mathrm{TiO}_{2}$ NPs and to minimize the disruption of the biofilm. ${ }^{34,42}$ The morphology of algal cells was checked after fixation (Fig. S-6B $\dagger$ ) showing most of the cells to have distorted membrane with a rough surface forming a network-like pattern. Similar structures formed by $C$. vulgaris were also reported by Iswarya et al. and Dalai et al. ${ }^{\mathbf{3 4 , 3 6}}$ However, round cells in a good shape are still clearly visible in the algal biofilm after fixation (Fig. S-6B $\dagger$ ).

The aim of ToF-SIMS experiment on C. vulgaris biofilm was not only to confirm the presence of $\mathrm{TiO}_{2}$ NPs but also to clarify their 3D distribution within this complex system. 3D analysis is implemented in ToF-SIMS by employing sputter ions to remove the uppermost layer of the sample after each analysis scan. This method allows for depth analyses to be conducted.

For the reconstruction of a real 3D structure, a homogeneous removal of sample layers by sputtering is needed. However, this is extremely difficult to implement on real organic systems because of their intrinsic heterogeneity even with an argon cluster ion beam. ${ }^{43}$ In fact, any highly packed dense agglomerates (e.g. storage vacuoles, chloroplasts, grana, etc.) and inorganic precipitates (e.g. intra- and extra-cellular phosphates, sulfates, etc.) will show a relatively lower sputter rate compared to other organic material. ${ }^{\mathbf{4 3 4 4}}$ Previous works from Houssiau group, reported how a low energy $\mathrm{Cs}^{+}$sputter source can moderate the difference in sputter rate for hybrid organic/ 
inorganic samples and preserve sensitive organics without causing significant damage..$^{43,45-48}$ Furthermore, implantation and deposition of Cs onto the sample surface are known to enhance the yield of negative secondary ions reducing their working function. ${ }^{43,47-50}$ Thus, the ToF-SIMS depth profiling of algal biofilm was performed with $500 \mathrm{eV} \mathrm{Cs}^{+}$ions as a sputtering projectiles.

In total, 777 data planes were acquired after 56 hours of analysis spent with almost complete sputtering of the biomass available in the analyzed $60 \times 60 \mu \mathrm{m}^{2}$ sample area. The 3D image of the analyzed sample volume (Fig. 5) shows a clear separation in three main compartments: (i) silicon substrate (grey) represented by ${ }^{28} \mathrm{Si}^{-}$ion counts; (ii) polymer coating layer (cyan) best pronounced in ${ }^{12} \mathrm{CH}_{2}{ }^{-}$secondary ion counts and (iii) C. vulgaris cells (green) identified mainly by the ${ }^{12} \mathrm{C}^{14} \mathrm{~N}^{-}$ion.

The $\mathrm{CN}^{-}(\mathrm{m} / \mathrm{z} 26)$ secondary ion distribution is commonly used in imaging SIMS experiments as an indicator of biomass and an intrinsic marker of spatial arrangement. ${ }^{10}$ The ion counts accumulated in mass peaks at $209 \mathrm{~m} / \mathrm{z}$ and $255 \mathrm{~m} / \mathrm{z}$ represent algal cell membrane and are shown in shades of green: light green and dark green respectively (Fig. 5). The $\mathrm{C}_{6} \mathrm{H}_{10} \mathrm{O}_{6} \mathrm{P}^{-}$secondary ion $(\mathrm{m} / \mathrm{z} 209)$ was ascribed to a fragment of phosphatidylglycerol (PG) arisen by the combined losses of both acid side chains. ${ }^{51,52}$ Indeed, the PG lipids are widely present in the membrane of $C$. vulgaris cells. ${ }^{53}$ The $\mathrm{C}_{16} \mathrm{H}_{31} \mathrm{O}_{2}{ }^{-}$ secondary ion $(\mathrm{m} / \mathrm{z} 255)$ has already been reported to originate from a lipid side chain of palmitic acid after deprotonation. ${ }^{15,54}$

Ti-containing secondary ions were identified in the mass spectrum by the clear isotopic pattern of titanium (refer to Fig. 4B and C). Since $\mathrm{TiO}_{2}$ NPs are well known to be biologically inactive and physiologically inert, ${ }^{55}$ all Ti-containing secondary ions were attributed to $\mathrm{TiO}_{2}$ NPs. Additional evidence for the association of mass peaks with $\mathrm{TiO}_{2} \mathrm{NPs}$ was acquired by comparing the mass spectra of $C$. vulgaris biofilm exposed to $\mathrm{TiO}_{2}$ NPs and non-exposed biofilm (Fig. S-7 $\dagger$ ). The composition of Ti-containing ionization products revealed a clear dependence on the molecular surrounding of $\mathrm{TiO}_{2} \mathrm{NPs}$ in different sample compartments (i.e. polymer layer, extracellular algal matrix, algae cell membrane). In fact, Fig. 5 shows different spatial distributions of $\mathrm{TiH}^{-}$( $m / z$ 49, yellow), $\mathrm{CsTi}^{-}(m / z$ 180.9; blue), $\mathrm{C}_{3} \mathrm{H}_{5} \mathrm{O}_{7} \mathrm{Ti}^{-}$( $m / z$ 201; purple) and $\mathrm{C}_{x} \mathrm{HTi}^{-}(\mathrm{m} / z \mathrm{73}, 97 ; x=$ 2,4 ; red) secondary ions. Separate ion distributions relatively to the algal cells are displayed in Fig. S-8. $\dagger$

The $\mathrm{TiH}^{-}$ion $(\mathrm{m} / z$ 49) (in yellow, Fig. 5 and S-8A $\dagger$ ) appeared to be the most abundant and common ionization product of $\mathrm{TiO}_{2}$ NPs in organics under the experimental conditions applied.

The $\mathrm{TiO}_{2}$ NPs distributed over the polymer coating layer are identified by $\mathrm{CsTi}^{-}$secondary ions formed due to the presence of Cs implanted into the uppermost sample layer upon sputtering..$^{50}$

The heaviest Ti-containing ion occurred at $201 \mathrm{~m} / \mathrm{z}$ has been interpreted as $\mathrm{C}_{3} \mathrm{H}_{5} \mathrm{O}_{7} \mathrm{Ti}^{-}$ions. Their $3 \mathrm{D}$ distribution (in purple, Fig. 5) is strictly correlated with the cell membrane and shows the $\mathrm{C}_{3} \mathrm{H}_{5} \mathrm{O}_{7} \mathrm{Ti}^{-}$products to originate from the interface between algal cells. The suggested chemical formula of this ion fits the composition of a hydrophilic lipid fragment $\left(\mathrm{C}_{3} \mathrm{H}_{5} \mathrm{O}_{3}\right.$ glycerol backbone) combined with $\mathrm{TiO}_{4}$. The ionization of $\mathrm{TiO}_{x}$ in such a large ion containing an organic fragment implies the extracellular adsorption of $\mathrm{TiO}_{2}$ NPs onto the lipid bilayer. The proximity of the NPs to the molecules defines the probability of organic fragments to be ionized in combination with $\mathrm{TiO}_{x}$. It is known that the inclusion of high-density phase (e.g. metal, oxide layers or NPs) enhances the yield of secondary ions from organic matrix..$^{56-60}$

The spatial distribution of the $\mathrm{C}_{2} \mathrm{HTi}^{-}$and $\mathrm{C}_{4} \mathrm{HTi}^{-}$ion yield were found to be similar and the ions counts in these mass peaks were therefore combined and are presented in red. These $\mathrm{C}_{x} \mathrm{HTi}^{-}$ions $(x=2,4)$ may be ascribed to $\mathrm{TiO}_{2}$ NPs associated with extracellular polymeric substance (EPS) surrounding the cells. However, it can be clearly seen that the areas presented in red are located exclusively at the cell side facing the $X$-direction whereas no $\mathrm{C}_{x} \mathrm{HTi}^{-}$secondary ions were detected on the opposite side (Fig. 5). Such a regular ion distribution seems to

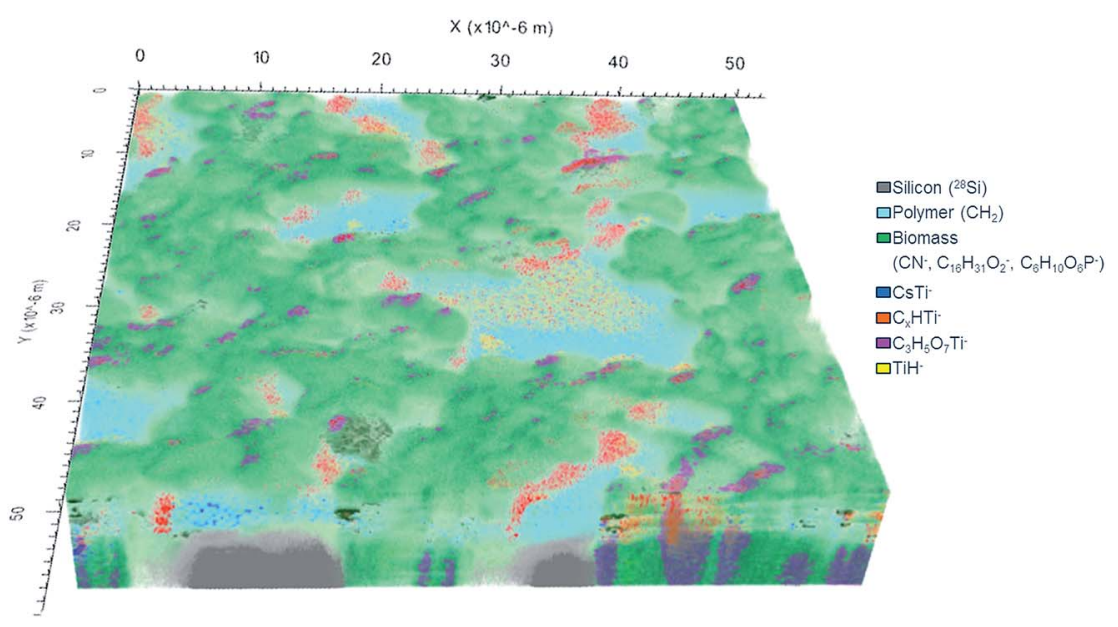

Fig. 5 3D reconstruction of ToF-SIMS data in overlay of the following ion signals: $\mathrm{Si}^{-}$(grey) to represent $\mathrm{Silicon}$ substrate, $\mathrm{CH}_{2}{ }^{-}$(cyan) to represent the polymer, $\mathrm{CN}^{-}, \mathrm{C}_{6} \mathrm{H}_{10} \mathrm{O}_{6} \mathrm{P}^{-}$and $\mathrm{C}_{16} \mathrm{H}_{31} \mathrm{O}_{2}^{-}$(shades of green) to display C. vulgaris cells and $\mathrm{TiH}^{-}$(yellow), $\mathrm{CsTi}^{-}$(blue), $\mathrm{C}_{3} \mathrm{H}_{5} \mathrm{O}_{7} \mathrm{Ti}^{-}$ (purple) and $\mathrm{C}_{x} \mathrm{HTi}^{-}\left(x=2,4\right.$ red) to render $\mathrm{TiO}_{2} \mathrm{NPs}$. 
be rather doubtful for a biological system and may originate from an experimental artifact. Shadowing and lateral displacement effects are observed in ToF-SIMS experiments on samples with pronounced surface topography and upon analysis within a large range of sample depths due to the $45^{\circ}$ angle mounting geometry of the primary ion source..$^{\mathbf{2 0 , 3 9 , 5 0}}$ These effects are revealed by the difference between values of lateral resolution derived from the right and left slopes on the Chessy standard structure (see Fig. $\mathrm{S}-5 \mathrm{~A} \uparrow$ for details). The algal cell height of $\sim 5$ $\mu \mathrm{m}$ may arise considerable lateral displacement artifacts in the visualization of samples with a large variation of depths. Therefore, components located on top and beneath the cell may appear laterally overlapping even though the imaged sample features are separated only in height. Thus, the considered $\mathrm{TiO}_{2}$ NPs yielding the $\mathrm{C}_{x} \mathrm{HTi}^{-}$secondary ions may be confined in a narrower space around the cells and not displaced in $X$ direction as it appears in the reconstructed 3D-image (further confirmation of this hypothesis is provided by Fig. S-8D and S$9 \dagger)$. These $\mathrm{TiO}_{2}$ enriched hetero-aggregates surrounding the cells can be formed due to the accumulation of NPs in the EPSinterface between the cell and the polymer coating upon sample shaking and cannot be ascribed to inherent cell physiology. It is worth to underline that the localization of $\mathrm{TiO}_{2}$ NPs was concluded from the detected Ti-containing secondary ions and not from the 3D image directly. The latter is used as a visual confirmation of the acquired spectrometry data.

The lateral resolution of the ToF-SIMS analysis on the algal biofilm (222 nm) was verified via a linescan over $\mathrm{PO}_{3}{ }^{-}$secondary ion map revealing sharp features of nano-sized phosphate precipitates (Fig. S-10†).

Amino group ionization products are commonly used in SIMS analysis for the identification of intracellular proteins. ${ }^{\mathbf{6 1 , 6 2}}$ Analysis of the mass spectrum integrated over the whole sample volume did not reveal any secondary ions comprising Ti and amino group. Even if this is due to a detection limit issue, the obtained results show the fraction of NPs internalized by algal cells to be considerably smaller than the NP fraction associated with the cell membrane and surrounding EPS. A possible explanation of a low uptake is the decrease of $\mathrm{TiO}_{2}$ NPs availability due to their agglomeration. The correlation between NP aggregation and decrease in their uptake has already been reported in other studies. ${ }^{36,63} \mathrm{NP}$ adhesion onto the cell membrane is one of the main mechanisms causing the toxicity of $\mathrm{TiO}_{2}$ NPs towards C. vulgaris. Their adhesion on the surface can cause the disruption of the cell membrane by clogging the pores and the channels limiting the uptake of nutrients. ${ }^{37,64}$ It has been reported previously that the NPs attached on the cell walls might serve as an adhesive agent between algae and cause this algal "flocculation" observed in Fig. S-6B. $\dagger^{35,37}$ Consequently, the light availability and production of chlorophyll may be also affected by the NPs aggregates formation., ${ }^{2,64}$

\section{Conclusions}

The simultaneous achievement of $108 \mathrm{~nm}$ lateral resolution together with an MRP over 5000 confirms ToF-SIMS to be a powerful tool to study nanoparticles-biota interaction allowing for the resolution of nano-sized phases and single microbial cells. The dependence of NP ionization products on the organic surroundings supports the elucidation of NP localization in complex host via analysis of secondary ions containing NP-related elements. The identification of relevant secondary ions becomes more consistent when a NP contains an element with a specific isotopic pattern reproduced in corresponding mass peaks. For a reliable high-resolution imaging of complex organic samples, sample preparation methods were developed to preserve the spatial distribution and chemical identity of NPs and host compartments. The imaging artifacts observed due to the $45^{\circ}$ primary ion beam incidence and the sample topography may be moderated by tilting the sample towards the normal incidence geometry. However, the application of delayed extraction mode provides reduced mass resolution (around 5000, MRP) and mass accuracy ( 100 ppm). Therefore, an unambiguous identification may not be obtained for every peak in the acquired mass spectra. Further improvement of ion beam focusing, mass resolving power and sensitivity of ToF-SIMS would expand its application into nanoscale research (e.g. molecular microbiology, nanomedicine, diagnostic) where a subcellular resolution and a single nanoparticle recognition is required.

\section{Conflicts of interest}

There are no conflicts to declare.

\section{Acknowledgements}

The funding of the ACEnano project within the European Union Horizon 2020 Program (H2020, grant agreement number 720952) is gratefully acknowledged. The authors appreciate the support of ProVIS Center for Chemical Microscopy by the Helmholtz Centre for Environmental Research - UFZ and the funds for ProVIS establishment provided by EFRE (Europäischer Fonds für regionale Entwicklung) and the federal state (Freistaat Sachsen).

\section{References}

1 F. Piccinno, F. Gottschalk, S. Seeger and B. Nowack, Industrial production quantities and uses of ten engineered nanomaterials in Europe and the world, $J$. Nanopart. Res., 2012, 14, 1109.

2 E. Navarro, F. Piccapietra, B. Wagner, F. Marconi, R. Kaegi, N. Odzak, L. Sigg and R. Behra, Toxicity of Silver Nanoparticles to Chlamydomonas reinhardtii, Environ. Sci. Technol., 2008, 42, 8959-8964.

3 L. Wang, L. Yan, J. Liu, C. Chen and Y. Zhao, Quantification of Nanomaterial/Nanomedicine Trafficking in Vivo, Anal. Chem., 2018, 90, 589-614.

4 J. W. Wills, H. D. Summers, N. Hondow, A. Sooresh, K. E. Meissner, P. A. White, P. Rees, A. Brown and S. H. Doak, Characterizing Nanoparticles in Biological Matrices: Tipping Points in Agglomeration State and Cellular Delivery in Vitro, ACS Nano, 2017, 11, 11986-12000. 
5 Y.-P. Kim, H. K. Shon, S. K. Shin and T. G. Lee, Probing nanoparticles and nanoparticle-conjugated biomolecules using time-of-flight secondary ion mass spectrometry, Mass Spectrom. Rev., 2015, 34, 237-247.

6 J. S. Fletcher, S. Rabbani, A. Henderson, P. Blenkinsopp, S. P. Thompson, N. P. Lockyer and J. C. Vickerman, A New Dynamic in Mass Spectral Imaging of Single Biological Cells, Anal. Chem., 2008, 80, 9058-9064.

7 J. S. Fletcher and J. C. Vickerman, A new SIMS paradigm for 2D and 3D molecular imaging of bio-systems, Anal. Bioanal. Chem., 2010, 396, 85-104.

8 M. K. Passarelli, A. Pirkl, R. Moellers, D. Grinfeld, F. Kollmer, R. Havelund, C. F. Newman, P. S. Marshall, H. Arlinghaus, M. R. Alexander, A. West, S. Horning, E. Niehuis, A. Makarov, C. T. Dollery and I. S. Gilmore, The 3D OrbiSIMS-label-free metabolic imaging with subcellular lateral resolution and high mass-resolving power, Nat. Methods, 2017, 14, 1175-1183.

9 M. Senoner and W. E. S. Unger, SIMS imaging of the nanoworld: applications in science and technology, J. Anal. At. Spectrom., 2012, 27, 1050-1068.

10 R. Sekine, K. L. Moore, M. Matzke, P. Vallotton, H. Jiang, G. M. Hughes, J. K. Kirby, E. Donner, C. R. M. Grovenor, C. Svendsen and E. Lombi, Complementary Imaging of Silver Nanoparticle Interactions with Green Algae: DarkField Microscopy, Electron Microscopy, and Nanoscale Secondary Ion Mass Spectrometry, ACS Nano, 2017, 11, 10894-10902.

11 A. Haase, H. F. Arlinghaus, J. Tentschert, H. Jungnickel, P. Graf, A. Mantion, F. Draude, S. Galla, J. Plendl, M. E. Goetz, A. Masic, W. Meier, A. F. Thünemann, A. Taubert and A. Luch, Application of laser postionization secondary neutral mass spectrometry/time-of-flight secondary ion mass spectrometry in nanotoxicology: Visualization of nanosilver in human macrophages and cellular responses, ACS Nano, 2011, 5, 3059-3068.

$12 \mathrm{~J}$. S. Fletcher, Latest applications of 3D ToF-SIMS bioimaging, Biointerphases, 2015, 10, 018902.

13 D. Touboul, F. Kollmer, E. Niehuis, A. Brunelle and O. Laprévote, Improvement of biological time-of-flightsecondary ion mass spectrometry imaging with a bismuth cluster ion source, J. Am. Soc. Mass Spectrom., 2005, 16, 1608-1618.

14 J. S. Fletcher, N. P. Lockyer, S. Vaidyanathan and J. C. Vickerman, TOF-SIMS 3D biomolecular imaging of Xenopus laevis oocytes using buckminsterfullerene (C60) primary ions, Anal. Chem., 2007, 79, 2199-2206.

15 Q. P. Vanbellingen, N. Elie, M. J. Eller, S. Della-Negra, D. Touboul and A. Brunelle, Time-of-flight secondary ion mass spectrometry imaging of biological samples with delayed extraction for high mass and high spatial resolutions, Rapid Commun. Mass Spectrom., 2015, 29, 1187-1195.

16 S. Wagner, A. Gondikas, E. Neubauer, T. Hofmann and F. Von Der Kammer, Spot the difference: Engineered and natural nanoparticles in the environment-release, behavior, and fate, Angew. Chem., Int. Ed., 2014, 53, 1239812419.

17 R. Kaegi, A. Voegelin, C. Ort, B. Sinnet, B. Thalmann, J. Krismer, H. Hagendorfer, M. Elumelu and E. Mueller, Fate and transformation of silver nanoparticles in urban wastewater systems, Water Res., 2013, 47, 3866-3877.

18 B. C. Reinsch, B. Forsberg, R. L. Penn, C. S. Kim and G. V. Lowry, Chemical Transformations during Aging of Zerovalent Iron Nanoparticles in the Presence of Common Groundwater Dissolved Constituents, Environ. Sci. Technol., 2010, 44, 3455-3461.

19 M. Bundschuh, J. Filser, S. Lüderwald, M. S. McKee, G. Metreveli, G. E. Schaumann, R. Schulz and S. Wagner, Nanoparticles in the environment: where do we come from, where do we go to?, Environ. Sci. Eur., 2018, 30, 6.

20 J. L. S. Lee, I. S. Gilmore, I. W. Fletcher and M. P. Seah, Topography and field effects in the quantitative analysis of conductive surfaces using ToF-SIMS, Appl. Surf. Sci., 2008, 255, 1560-1563.

21 L. Veith, D. Dietrich, A. Vennemann, D. Breitenstein, C. Engelhard, U. Karst, M. Sperling, M. Wiemann and B. Hagenhoff, Combination of micro X-ray fluorescence spectroscopy and time-of-flight secondary ion mass spectrometry imaging for the marker-free detection of $\mathrm{CeO}_{2}$ nanoparticles in tissue sections, J. Anal. At. Spectrom., 2018, 33, 491-501.

$22 \mathrm{H}$. Stryhanyuk, Vorrichtung und Verfahren zur submolekularen Trockenreinigung und/oder zur Hydrophilisierung der Oberfläche von Festkörpern, EU Patent 18179 810.9, 2018.

23 J. R. Vig, UV/ozone cleaning of surfaces, J. Vac. Sci. Technol., A, 1985, 3, 1027-1034.

24 H. W. Nichols and H. C. Bold, Trichosarcina polymorpha Gen. et Sp. Nov., J. Phycol., 1965, 1, 34-38.

25 N. R. Moheimani, M. A. Borowitzka, A. Isdepsky and S. F. Sing, in Algae for Biofuels and Energy, Springer Netherlands, Dordrecht, 2013, pp. 265-284.

26 R. D. Handy, G. Cornelis, T. Fernandes, O. Tsyusko, A. Decho, T. Sabo-Attwood, C. Metcalfe, J. A. Steevens, S. J. Klaine, A. A. Koelmans and N. Horne, Ecotoxicity test methods for engineered nanomaterials: Practical experiences and recommendations from the bench, Environ. Toxicol. Chem., 2012, 31, 15-31.

27 K. L. Mittal, International Symposium on Polymer Surface Modification: relevance to adhesion, Polymer surface modification. Volume 3: relevance to adhesion, VSP, 2004.

28 S. Berkebile and J. Gaier, in 42nd International Conference on Environmental Systems, American Institute of Aeronautics and Astronautics, Reston, Virginia, 2012, pp. 1-10.

29 J. B. Hu, Y. C. Chen and P. L. Urban, Coffee-ring effects in laser desorption/ionization mass spectrometry, Anal. Chim. Acta, 2013, 766, 77-82.

$30 \mathrm{H}$. Hu and R. G. Larson, Marangoni effect reverses coffeering depositions, J. Phys. Chem. B, 2006, 110, 7090-7094.

31 A. L. M. Marsico, B. Duncan, R. F. Landis, G. Y. Tonga, V. M. Rotello and R. W. Vachet, Enhanced Laser Desorption/Ionization Mass Spectrometric Detection of 
Biomolecules Using Gold Nanoparticles, Matrix, and the Coffee Ring Effect, Anal. Chem., 2017, 89, 3009-3014.

32 R. D. Deegan, O. Bakajin, T. F. Dupont, G. Huber, S. R. Nagel and T. A. Witten, Capillary flow as the cause of ring stains from dried liquid drops, Nature, 1997, 389, 827-829.

33 P. D. Christy, N. S. N. Jothi, N. Melikechi and P. Sagayaraj, Synthesis, structural and optical properties of well dispersed anatase $\mathrm{TiO}_{2}$ nanoparticles by non-hydrothermal method, Cryst. Res. Technol., 2009, 44, 484-488.

34 S. Dalai, S. Pakrashi, M. J. Nirmala, A. Chaudhri, N. Chandrasekaran, A. B. Mandal and A. Mukherjee, Cytotoxicity of $\mathrm{TiO}_{2}$ nanoparticles and their detoxification in a freshwater system, Aquat. Toxicol., 2013, 138-139, 1-11.

35 I. M. Sadiq, S. Dalai, N. Chandrasekaran and A. Mukherjee, Corrigendum to "Ecotoxicity study of titania (TiO2) NPs on two microalgae species: Scenedesmus sp. and Chlorella sp.”, Ecotoxicol. Environ. Saf., 2017, 142, 597.

36 V. Iswarya, M. Bhuvaneshwari, S. A. Alex, S. Iyer, G. Chaudhuri, P. T. Chandrasekaran, G. M. Bhalerao, S. Chakravarty, A. M. Raichur, N. Chandrasekaran and A. Mukherjee, Combined toxicity of two crystalline phases (anatase and rutile) of Titania nanoparticles towards freshwater microalgae: Chlorella sp, Aquat. Toxicol., 2015, 161, 154-169.

37 A. Oukarroum, S. Bras, F. Perreault and R. Popovic, Inhibitory effects of silver nanoparticles in two green algae, Chlorella vulgaris and Dunaliella tertiolecta, Ecotoxicol. Environ. Saf., 2012, 78, 80-85.

38 K. Van Hoecke, K. A. C. De Schamphelaere, P. Van der Meeren, S. Lucas and C. R. Janssen, Ecotoxicity of silica nanoparticles to the green alga Pseudokirchneriella subcapitata: importance of surface area, Environ. Toxicol. Chem., 2008, 27, 1948-1957.

39 S. Rangarajan and B. J. Tyler, Topography in secondary ion mass spectroscopy images, Vacuum, 2006, 1730, 1730-1736.

40 C. Bich, R. Havelund, R. Moellers, D. Touboul, F. Kollmer, E. Niehuis, I. S. Gilmore and A. Brunelle, Argon cluster ion source evaluation on lipid standards and rat brain tissue samples, Anal. Chem., 2013, 85, 7745-7752.

41 OECD, Freshwater alga and cyanobacteria, growth inhibition test, Test Guideline 201, Oecd Guidel. Test. Chem., 2011, pp.126.

42 A. Worrich, H. Stryhanyuk, N. Musat, S. König, T. Banitz, F. Centler, K. Frank, M. Thullner, H. Harms, H.-H. Richnow, A. Miltner, M. Kästner and L. Y. Wick, Mycelium-mediated transfer of water and nutrients stimulates bacterial activity in dry and oligotrophic environments, Nat. Commun., 2017, 8, 15472.

43 C. Noël and L. Houssiau, Hybrid Organic/Inorganic Materials Depth Profiling Using Low Energy Cesium Ions, J. Am. Soc. Mass Spectrom., 2016, 27, 908-916.

44 M. P. Seah, Universal Equation for Argon Gas Cluster Sputtering Yields, J. Phys. Chem. C, 2013, 117, 12622-12632.

45 L. Houssiau and N. Mine, Molecular depth profiling of polymers with very low energy reactive ions, Surf. Interface Anal., 2010, 42, 1402-1408.
46 L. Houssiau and N. Mine, Molecular depth profiling with reactive ions, or why chemistry matters in sputtering, Surf. Interface Anal., 2011, 43, 146-150.

$47 \mathrm{~N}$. Wehbe and L. Houssiau, Comparative study of the usefulness of low energy $\mathrm{Cs}+, \mathrm{Xe}+$, and $\mathrm{O} 2+$ ions for depth profiling amino-acid and sugar films, Anal. Chem., 2010, 82, 10052-10059.

48 N. Wehbe, J. Pireaux and L. Houssiau, XPS Evidence for Negative Ion Formation in SIMS Depth Profiling of Organic Material with Cesium, J. Phys. Chem. C, 2014, 118, 2661326620.

49 H. G. Cramer, T. Grehl, F. Kollmer, R. Moellers, E. Niehuis and D. Rading, Depth profiling of organic materials using improved ion beam conditions, Appl. Surf. Sci., 2008, 255, 966-969.

50 E. Niehuis, T. Grehl, F. Kollmer, R. Moellers, D. Rading, R. Kersting and B. Hagenhoff, MCs+ depth profiling using cluster primary ions, Surf. Interface Anal., 2011, 43, 204-206.

51 F.-F. Hsu and J. Turk, Studies on phosphatidylglycerol with triple quadrupole tandem mass spectrometry with electrospray ionization: Fragmentation processes and structural characterization, J. Am. Soc. Mass Spectrom., 2001, 12, 1036-1043.

52 F. Hsu, J. Turk, T. D. Williams and R. Welti, Electrospray ionization multiple stage quadrupole ion-trap and tandem quadrupole mass spectrometric studies on phosphatidylglycerol from arabidopsis leaves, J. Am. Soc. Mass Spectrom., 2007, 18, 783-790.

53 C. Zuñiga, C.-T. Li, T. Huelsman, J. Levering, D. C. Zielinski, B. O. McConnell, C. P. Long, E. P. Knoshaug, M. T. Guarnieri, M. R. Antoniewicz, M. J. Betenbaugh and K. Zengler, Genome-Scale Metabolic Model for the Green Alga Chlorella vulgaris UTEX 395 Accurately Predicts Phenotypes under Autotrophic, Heterotrophic, and Mixotrophic Growth Conditions, Plant Physiol., 2016, 172, 589-602.

54 K. Schaepe, D. R. Bhandari, J. Werner, A. Henss, A. Pirkl, M. Kleine-Boymann, M. Rohnke, S. Wenisch, E. Neumann, J. Janek and B. Spengler, Imaging of Lipids in Native Human Bone Sections Using TOF-Secondary Ion Mass Spectrometry, Atmospheric Pressure Scanning Microprobe Matrix-Assisted Laser Desorption/Ionization Orbitrap Mass Spectrometry, and Orbitrap-Secondary Ion Mass Spectrometry, Anal. Chem., 2018, 90, 8856-8864.

55 H. Shi, R. Magaye, V. Castranova and J. Zhao, Titanium dioxide nanoparticles: a review of current toxicological data, Part. Fibre Toxicol., 2013, 10, 15.

56 A. Delcorte, S. Yunus, N. Wehbe, N. Nieuwjaer, C. Poleunis, A. Felten, L. Houssiau, J. J. Pireaux and P. Bertrand, Metalassisted secondary ion mass spectrometry using atomic (Ga+, In+) and fullerene projectiles, Anal. Chem., 2007, 79, 3673-3689.

57 A. Heile, D. Lipinsky, N. Wehbe, A. Delcorte, P. Bertrand, A. Felten, L. Houssiau, J. J. Pireaux, R. De Mondt, L. Van Vaeck and H. F. Arlinghaus, Metal-assisted SIMS and cluster ion bombardment for ion yield enhancement, Appl. Surf. Sci., 2008, 255, 941-943. 
58 O. Restrepo, A. Prabhakaran, K. Hamraoui, N. Wehbe, S. Yunus, P. Bertrand and A. Delcorte, Mechanisms of metal-assisted secondary ion mass spectrometry: A mixed theoretical and experimental study, Surf. Interface Anal., 2010, 42, 1030-1034.

59 A. Delcorte, J. Bour, F. Aubriet, J. F. Muller and P. Bertrand, Sample Metallization for Performance Improvement in Desorption/Ionization of Kilodalton Molecules: Quantitative Evaluation, Imaging Secondary Ion MS, and Laser Ablation, Anal. Chem., 2003, 75, 6875-6885.

60 A. Gulin, M. Mochalova, N. Denisov and V. Nadtochenko, Secondary ion mass spectrometric signal enhancement of phosphatidylcholine dioleoyl on enlarged nanoparticles surface, Appl. Surf. Sci., 2014, 316, 36-41.

61 D. Breitenstein, C. E. Rommel, R. Möllers, J. Wegener and B. Hagenhoff, The chemical composition of animal cells and their intracellular compartments reconstructed from 3D mass spectrometry, Angew. Chem., Int. Ed., 2007, 46, 5332-5335.

62 J.-L. Guerquin-Kern, T.-D. Wu, C. Quintana and A. Croisy, Progress in analytical imaging of the cell by dynamic secondary ion mass spectrometry (SIMS microscopy), Biochim. Biophys. Acta, Gen. Subj., 2005, 1724, 228-238.

63 B. Ekstrand-Hammarström, C. M. Akfur, P. O. Andersson, C. Lejon, L. Österlund and A. Bucht, Human primary bronchial epithelial cells respond differently to titanium dioxide nanoparticles than the lung epithelial cell lines A549 and BEAS-2B, Nanotoxicology, 2012, 6, 623-634.

64 C. Wei, Y. Zhang, J. Guo, B. Han, X. Yang and J. Yuan, Effects of silica nanoparticles on growth and photosynthetic pigment contents of Scenedesmus obliquus, J. Environ. Sci., 2010, 22, 155-160. 